\title{
Star Formation in a Multi-Phase ISM
}

\author{
Stefan Harfst, Christian Theis and Gerhard Hensler \\ Institute for Theoretical Physics and Astrophysics, University Kiel, Germany
}

\begin{abstract}
We present a 3d code for the dynamical evolution of a multi-phase interstellar medium (ISM) coupled to stars via star formation (SF) and feedback processes. The multi-phase ISM consists of clouds (sticky particles) and diffuse gas $(\mathrm{SPH})$ : exchange of matter, energy and momentum is achieved by drag (due to ram pressure) and condensation or evaporation processes. The cycle of matter is completed by SF and feedback by SNe and PNe. A SF scheme based on a variable SF efficiency as proposed by Elmegreen \& Efremov (1997) is presented. For a Milky Way type galaxy we get a SF rate of $\sim 1 \mathrm{M}_{\odot} \mathrm{yr}^{-1}$ with an average SF efficiency of $\sim 5 \%$.
\end{abstract}

Keywords: Methods: N-body simulations, Galaxies: evolution, Galaxies: ISM

\section{Introduction}

So far in 3d models of galaxies the ISM is mostly described either as a diffuse phase by SPH (e.g. Hernquist \& Katz, 1989) or as a clumpy phase by sticky particles (e.g. Theis \& Hensler, 1993). Alternatively, chemo-dynamical models use a multi-phase ISM, but were usually restricted due to spherical or axisymmetric systems (e.g. Theis et al., 1992; Samland et al., 1997). In order to extend the chemodynamical models to three dimensions we combine both treatments in a particle based code: the hot diffuse gas phase is described by a SPH formalism, whereas the cold molecular clouds are represented by sticky particles. The coupling between the gaseous phases is achieved by condensation/evaporation $(\mathrm{C} / \mathrm{E})$ and by a drag force due to ram pressure. Energy is dissipated by cloud-cloud-collisions or by radiative cooling. Furthermore, stars are formed in clouds and the stars return mass and energy to the gas by feedback processes (supernovae, planetary nebulae).

In $\mathrm{SPH}$ codes the $\mathrm{SF}$ is generally based on the Schmidt law, i.e. the SF rate depends on gas density and a characteristic time scale. For sticky particles the SF can be coupled to cloud-cloud collisions, or a single cloud forms stars with a constant SF efficiency. Here, a $\mathrm{SF}$ scheme using a different approach is presented: the process of SF is treated individually for each cloud. The SF efficiency is dependent on local properties of the ISM and the star forming clouds, thereby enabling self-regulation of SF by feedback.

(c) 2019 Kluwer Academic Publishers. Printed in the Netherlands. 


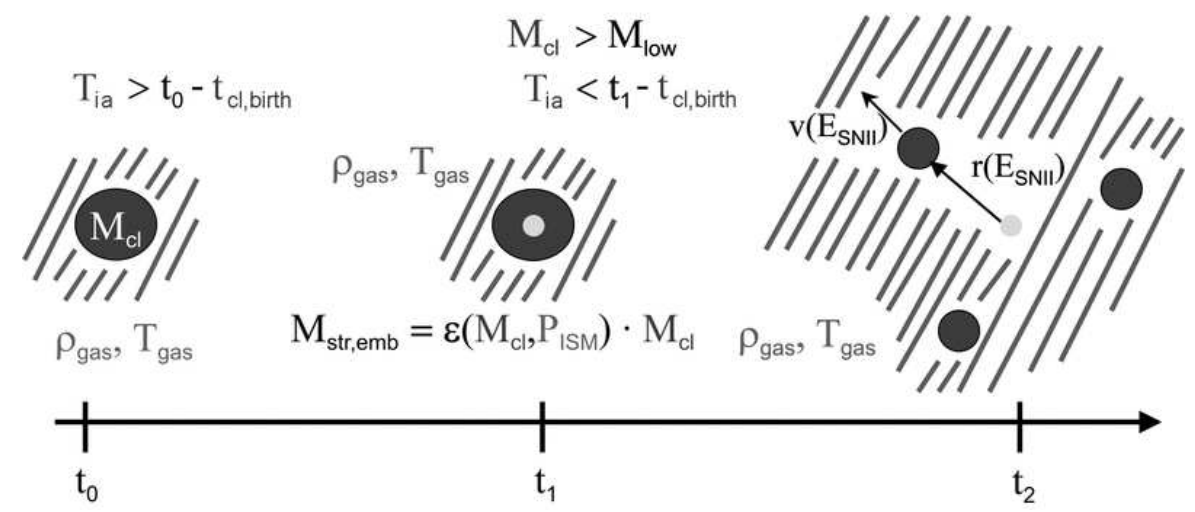

Figure 1. The SF scheme (see text for details)

\section{Numerical Treatment}

The gravitational force for all particles is calculated with a TREE algorithm. A dark matter halo can be modeled as an external, static potential (static halo) or by means of particles (life halo). The integration of the system is done with a leap-frog scheme. Individual time steps are used for each particle.

The drag force and the mass exchange rates for $\mathrm{C} / \mathrm{E}$ are calculated following Cowie et al. (1981) using a mass-radius-relation for the clouds based on observations (e.g. Rivolo \& Solomon, 1988). Effects of both processes are individually determined for each cloud using the local density, temperature and velocity of the hot gas. Energy can be dissipated by inelastic cloud-cloud collisions (Theis \& Hensler, 1993) and by radiative cooling (Böhringer \& Hensler, 1989).

Clouds can transform some of their mass into new stars. In Fig. 1 this process is drawn schematically. Each cloud is inactive for a given time $T_{i a}\left(t_{0}\right)$. An embedded star cluster is then formed $\left(t_{1}\right)$ with the $S F$ efficiency $\epsilon$ being a function of cloud mass and gas pressure according to Elmegreen \& Efremov (1997). The energy released by SNe is calculated using a multi-power law IMF (Kroupa et al., 1993). This energy injection disrupts the cloud into smaller fragments $\left(t_{2}\right)$. The time for disruption $T_{\text {dis }}\left(t_{2}-t_{1}\right.$ in Fig. 1$)$ is determined from the energy input: a self-similar solution is used to calculate the SN shell expansion. It is assumed that the cloud disrupts when the shell radius equals the cloud radius. The fragments are then placed symmetrically on the shell with velocities corresponding to the expansion velocity at $\mathrm{T}_{\text {dis }}$. The energy not used for cloud disruption and the mass ejected by SNe is returned to the hot gas phase (SPH particles). Additionally, the mass returned by $\mathrm{PNe}$ is added to surrounding cloud particles. 


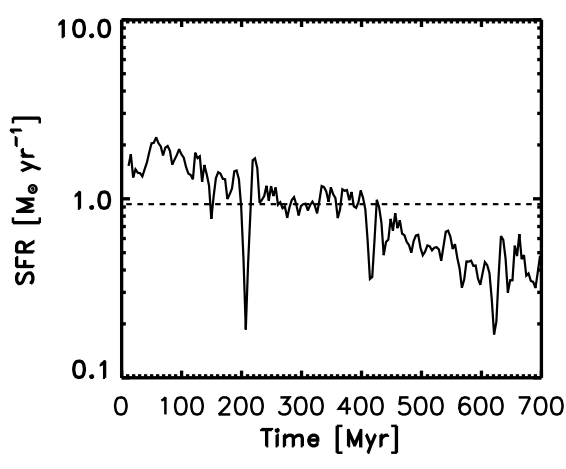

a) Star formation rate

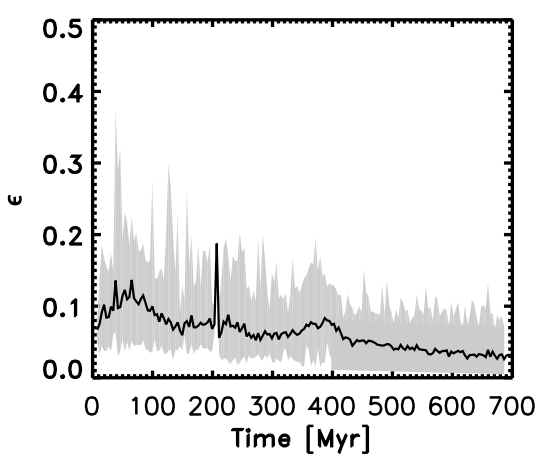

b) Star formation efficiency

Figure 2. In the left plot the temporal evolution (solid line) and the average (dashed line) of the SF rate are shown for a Milky Way type galaxy. The right plot shows the average SF efficiency (solid line) and the range of individual SF efficiencies (shaded area) with time for the same model.

\section{The model and results}

Initially a galaxy similar to the Milky Way was set up using the galaxy building package described by Kuijken \& Dubinski (1995). A cloudy gas phase is added by treating every tenth particle from the stellar disk as a cloud. The total mass in clouds is $\mathrm{M}_{\mathrm{cl} \text {,tot }} \approx 3.4 \cdot 10^{9} \mathrm{M}_{\odot}$ and each cloud is assigned a time of inactivity $\mathrm{T}_{\mathrm{ia}}$ between 0 and $200 \mathrm{Myr}$. Finally, a hot gas halo with a total mass of $\mathrm{M}_{\text {hot,tot }} \approx 2 \cdot 10^{8} \mathrm{M}_{\odot}$ is added, which is designed to be in hydrostatic equilibrium in the halo potential.

In Fig. 2a the resulting SF rate is shown, which is on average $\sim 1 \mathrm{M}_{\odot} \mathrm{yr}^{-1}$. The SF rate is slowly decreasing with time due to 1) the decreasing mass in the cloudy phase of the ISM and 2) the decreasing average SF efficiency (Fig. 2b). The decrease of $\epsilon$ is correlated with a decreasing $\mathrm{M}_{\mathrm{cl}}$ as expected from Elmegreen \& Efremov (1997). After $700 \mathrm{Myr}$ the average SF efficiency is less than $5 \%$ and the maximum efficiency is always lower than $20 \%$. From such a low SF efficiency we do not expect any non-induced globular cluster formation in the disk.

In Fig. 3a the mass return rate due to stellar death is shown: the $\mathrm{SNe}$ rate is about $10 \%$ of the SF rate and in agreement with the mass fraction of massive stars. The mass return rate by $\mathrm{PNe}$ is approximately equal to the SF rate due to a contribution of older stars in the initial model.

Condensation and evaporation rates are shown in Fig 3b. The evaporation rate is about 10 times higher than the condensation rate because 


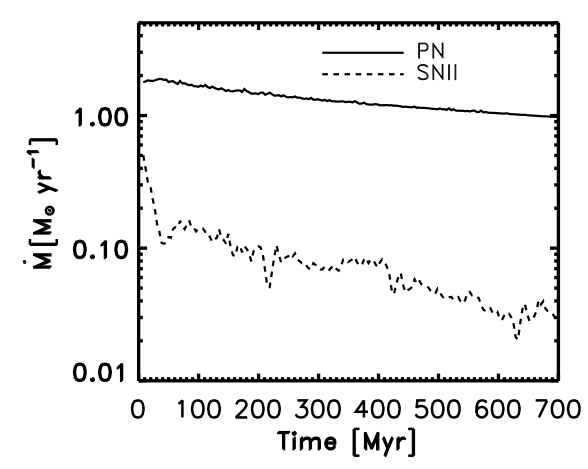

a) Feedback

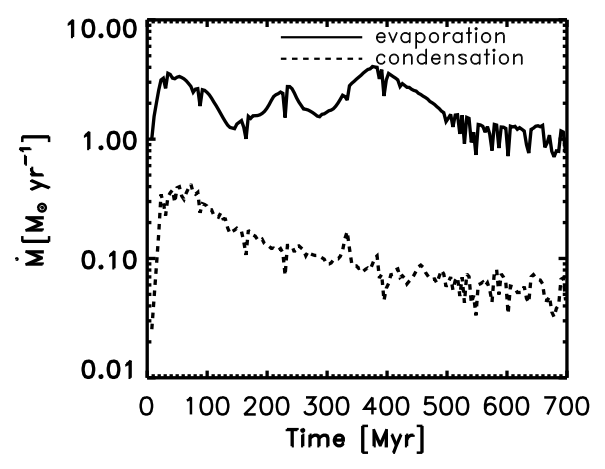

b) Condensation and evaporation

Figure 3. Mass flow rates. The left plot shows the temporal evolution of the mass return rate by $\mathrm{PNe}$ (solid) and $\mathrm{SNe}$ (dashed). In the right plot condensation (dashed) and evaporation (solid) rates are shown.

the temperature of the hot gas amounts to several $10^{6} \mathrm{~K}$. While the SF establishes an equilibrium on a rather short time scale $\mathrm{C} / \mathrm{E}$ have not reached an equilibrium state after $700 \mathrm{Myr}$. This is in agreement with the analytic work of Köppen et al. (1998). They predict an equilibrium state only after a few condensation time scales of evolution, which is a few Gyr in this model.

\section{Acknowledgements}

This work is supported by the Deutsche Forschungsgemeinschaft (DFG) under the grant TH-511/2-3.

\section{References}

Böhringer, H. and Hensler, G.: 1989. A\&A A 215, 147.

Cowie, L. L., McKee, C. F., and Ostriker, J. P.: 1981. ApJ 247, 908.

Elmegreen, B. G. and Efremov, Y. N.: 1997. ApJ 480, 235.

Hernquist, L. and Katz, N.: 1989. ApJSS 70, 419.

Köppen, J., Theis, C., and Hensler, G.: 1998. A\&A 331, 524.

Kroupa, P., Tout, C. A., and Gilmore, G.: 1993. MNRAS 262, 545.

Kuijken, K. and Dubinski, J.: 1995. MNRAS 277, 1341.

Rivolo, A. R. and Solomon, P. M.: 1988. In: R. L. Dickman, R. L. Snell, and J. S.

Young (eds.): Molecular Clouds in the Milky Way and External Galaxies. p. 42.

Samland, M., Hensler, G., and Theis, C.: 1997. ApJ 476, 544.

Theis, C., Burkert, A., and Hensler, G.: 1992. A\&A 265, 465.

Theis, C. and Hensler, G.: 1993. A\&A 280, 85. 